\title{
Membrane Potential Changes in Sphincter Motoneurons during Micturition in the Decerebrate Cat
}

\author{
B. Fedirchuk and S. J. Shefchyk \\ Departments of Medicine and of Physiology, University of Manitoba, Winnipeg, Canada R3E OW3
}

\begin{abstract}
Intracellular recordings from external urethral sphincter (EUS) and external anal sphincter (EAS) motoneurons were obtained during micturition in the decerebrate cat. The neural circuitry mediating micturition was activated by distension of the bladder or by electrical stimulation of the pontine micturition center (PMC). During micturition, the membrane potential of EUS motoneurons hyperpolarized 3-9 $\mathrm{mV}$, during which time the motoneuron somatic membrane conductance increased. The membrane hyperpolarization could be reversed with passive diffusion or active ejection of chloride from the intracellular microelectrode into the motoneuron. In contrast, the membrane potential of EAS motoneurons either depolarized slightly or showed no change during micturition. We have shown that the neural circuitry mediating micturition can influence the EUS and EAS independently. In addition, stimulation of the PMC provides a valuable tool for identifying spinal neurons mediating the postsynaptic inhibition of EUS motoneurons during micturition.
\end{abstract}

[Key words: input resistance, sacral, pudendal, micturition, urethra, bladder]

The skeletal muscles of the external urethral sphincter (EUS) and external anal sphincter (EAS) are important in the maintenance of urinary and fecal continence and in facilitating the cxpulsion phase of micturition and defecation. Inappropriate activation or relaxation of these sphincters results in significant clinical dysfunction (for reviews, see DeGroat, 1990; Dubrovsky and Filipini, 1990). Both striated sphincter muscles are innervated by $\alpha$-motoneurons located in the ventral horn of the sacral spinal cord. Collectively, these cells are commonly called pudendal or sphincter motoneurons and are often referred to as Onur's nucleus. Studies using intracellular recordings from single sphincter motoneurons have shown that EUS and EAS motoneurons have comparable membrane electrical properties (Hochman et al., 1991; Sasaki, 1991) and similar segmental and supraspinal synaptic inputs (Bradley and Teague, 1977; Mackel, 1979; Fedirchuk et al., 1990, 1992). During micturition, EUS activity decreases while activity in the EAS can remain or increase (Fedirchuk and Shefchyk, 1991; see also Dubrovsky and

Received Oct. 19, 1992; revised Jan. 15, 1993; accepted Feb. 3, 1993.

We thank Sharon McCartney and Maria Setterbom for their unfailing assistance in this work and Dr. David McCrea and the journal reviewers for thcir hclpful comments. This work was funded by a Medical Research Council (MRC) of Canada operating grant to S.J.S.; S.J.S. is an MRC scholar; B.F. has an MRC studentship.

Correspondence should be addressed to Dr. S. Shefchyk, Department of Physiology, University of Manitoba, 770 Bannatyne Avenue, Winnipeg, Canada R3E ow3.

Copyright (c) 1993 Society for Neuroscience $0270-6474 / 93 / 133090-05 \$ 05.00 / 0$
Filipini, 1990). The suppression of EUS activity during micturition could be explained by a direct inhibitory synaptic input on the motoneurons or actions at a premotoneuronal site (i.e., primary afferents or interneurons). Intracellular recording from these motoneurons during micturition can examine this question directly.

The inherent slowness of producing a distension-evoked reflex void by filling the bladder with fluid increases the difficulty of experiments in which one seeks to record intracellularly from small spinal neurons. Thus, the present experiments use both bladder infusion and electrical stimulation of the pontine micturition center (PMC) (Sugaya et al., 1987; Noto et al., 1989; Shefchyk, 1989; Kruse et al., 1990; Fedirchuk and Shefchyk, 1991; Mallory et al., 1991) to evoke micturition during intracellular recordings from EUS and EAS motoneurons.

Portions of these data have becn presented in abstract form (Fedirchuk et al., 1990, 1991).

\section{Materials and Methods}

Data were obtained from 17 precollicular postmammillary decerebrate male cats weighing between 2.2 and $3.8 \mathrm{~kg}$. Halothane delivered in a mixture of oxygen and nitrous oxide was used to induce ( $5 \%$ halothane) and maintain anesthesia (0.8-1.5\%) throughout the surgery until the decerebration was completed. General surgical procedures and maintenance of the animal are described by Fedirchuk et al. (1992). A lateral incision at the base of the tail exposed the ischio-rectal cavity. The exposed branches of the ipsilateral pudendal nerve innervating the EUS and EAS muscles were separated from surrounding tissue, cut, and placed on silver bipolar electrodes that could be used for either stimulating or recording. Laminectomy exposed the L6-S3 spinal cord, and the animal was placed in a spinal cord recording frame.

Micturition was evoked in one of two ways. In 13 animals, a saline solution $\left(0.9 \% \mathrm{NaCl}, 35^{\circ} \mathrm{C}, 2 \mathrm{ml} / \mathrm{min}\right)$ was infused into the bladder via the suprapubic catheter until the micturition threshold volume was reached $(7-27 \mathrm{ml})$. At the threshold volume, the bladder pressure would increase and the EUS electroneurographic (ENG) activity would decrease, during which time fluid in the bladder was expelled in a continuous stream. In four cats the bladder neck was ligated and infusion of fluid into the bladder continued until periodic isometric bladder contractions began. Infusion was then stopped and the periodic contractions continued as long as the volume in the bladder was maintained. The peak pressure during each of these isometric micturition contractions was comparable to, or greater than, that observed in the freely voiding preparations. During each isometric contraction the EUS ENG activity decreased.

The second method for evoking micturition used electrical stimulation of the PMC (see Fedirchuk and Shefchyk, 1991). In six cats a monopolar electrode was positioned in the rostral pons at a site that upon stimulation (100-200 $\mu \mathrm{A}, 100 \mathrm{~Hz}, 0.2 \mathrm{msec}$ square pulse) produced a bladder pressure increase coordinated with a decrease in EUS ENG activity during bladder emptying (for methods, refer to Shefchyk, 1989; Fedirchuk and Shefchyk, 1991). Preceding the electrical stimulation, a volume of warm saline approximately $50 \%$ of that required to elicit a distension reflex void was infused into the bladder.

Intracellular records were obtained using glass microelectrodes filled 


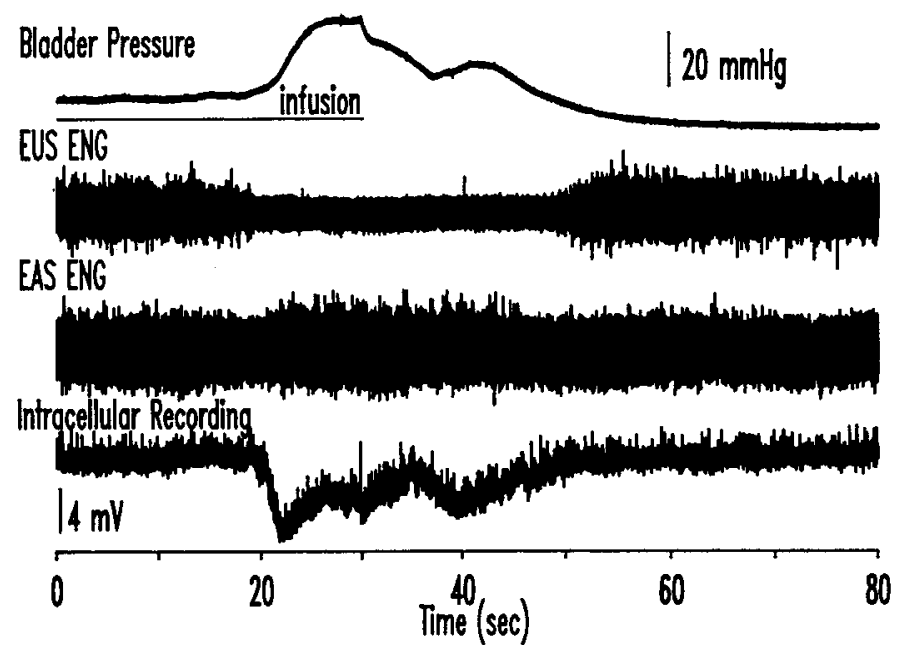

Figure 1. Intracellular recording from an EUS motoneuron during distension-evoked voiding (resting $E_{m},-60 \mathrm{mV}$ ).

with $2 \mathrm{M}$ potassium citrate or $2 \mathrm{M}$ potassium chloride (tip diameter, $1.6-1.8 \mu \mathrm{m}$; resistance, $2-5 \mathrm{M} \Omega$ ). Motoneurons were impaled in the S1 spinal segment and identified by antidromic activation from electrical stimulation of either the EUS or EAS branch of the pudendal nerve. In seven motoneurons rectangular hyperpolarizing current pulses $(500 \mu \mathrm{sec}$ in duration, 1-7 nA in amplitude) were injected into the cell at rates of $2.5-7 \mathrm{~Hz}$ throughout the voiding cycle to assess motoneuron conductance (see Carlen et al., 1980). These current pulses produced voltage deflections ranging from 0.5 to $2 \mathrm{mV}$ in amplitude. In three cells, we injected continuous hyperpolarizing current through the electrode in addition to current pulses in the absence of micturition. This was done to examine if hyperpolarization of the motoneuron membrane activated membrane currents (i.e., a subthreshold rectifying current; see Hochman et al., 1991; Sasaki, 1991) that could have contributed to the changes in conductance detected during micturition.

The bladder pressure, ENGs, stimulus markers, and a monitor of the intracellular current injection were digitized and stored on computer (Masscomp 5400). The low-gain and high-gain intracellular microelectrode records were digitized at rates of 5 and $15 \mathrm{kHz}$, respectively. In trials where motoneuron conductance was assessed, a portion of the microelectrode recording (30-80 msec duration) starting prior to and including the injected current pulse was stored on computer for off-line averaging. Each trial typically contained records from the prevoid, voiding, and postvoid periods. We assessed changes in motoneuron conductance by dividing the voiding cycle into $5-20$ equal, contiguous time scgments and comparing the averaged voltage deflections produced by short current pulse injection during these different times.

\section{Results}

Intracellular recordings were obtained from 30 EUS and 6 EAS motoneurons with resting membrane potentials of -50 to -60 $\mathrm{mV}$. All EUS motoneurons monitored during distension-evoked bladder contractions $(n=25)$ displayed a characteristic membrane hyperpolarization during the micturition reflex (Fig. 1). Unlike Shimoda et al. (1992), we did not observe bursts of activity in the EUS ENG or in EUS motoneurons before or during voiding. This may be explained by the fact that Shimoda et al. (1992) used rapid filling of the bladder (6-18 times faster than in the present study) to evoke micturition and this appeared to have evoked additional sphincter guarding reflexes.

In 5 of 25 EUS motoneurons examined during distensionevoked voiding, a small gradual hyperpolarization $(2-5 \mathrm{mV}$, mean $3.2 \mathrm{mV}$ ) of the motoneuron membrane potential preceded micturition (see also Shimoda et al., 1992). As the bladder started to contract, there was an additional rapid hyperpolarization associated with the micturition. The amplitude of this rapid

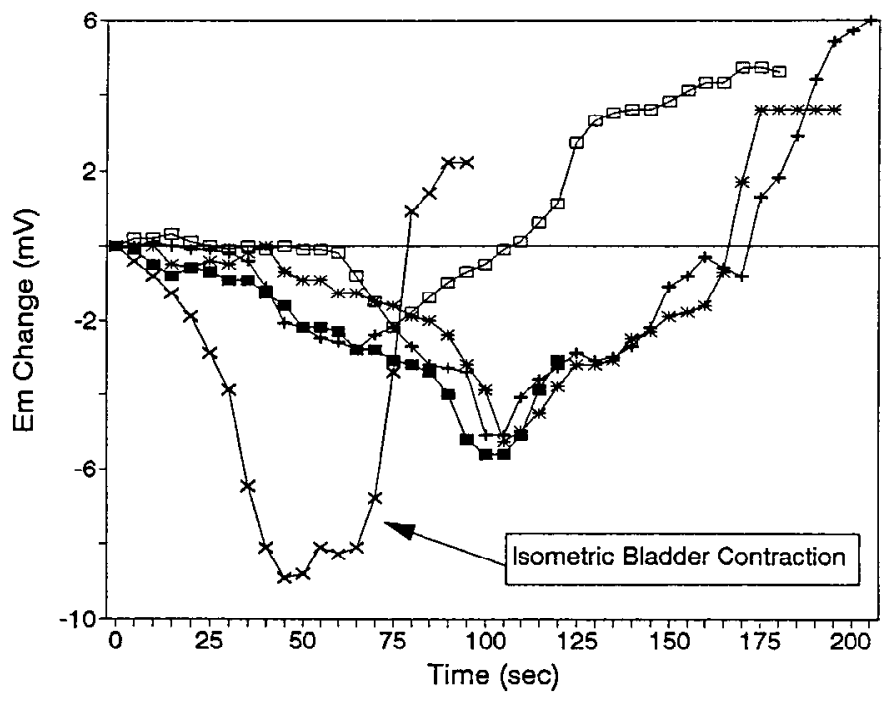

Figure 2. The time course and magnitude of membrane potential changes of five EUS motoneurons (each represented by a different symbol) during micturition. EUS motoneurons hyperpolarized during the period of bladder contraction and reduction of EUS ENG activity associated with distension-evoked micturition. The values within any trial are normalized to the prevoid membrane potential value for that trial.

membrane hyperpolarization, which was observed in all $25 \mathrm{EUS}$ motoneurons, ranged from 3 to $9 \mathrm{mV}$ (mean $5.4 \mathrm{mV}$ ) compared to the membrane potential immediately preceding the void.

In five motoneurons the membrane potential gradually depolarized due to a slight deterioration in the recording over the course of several conseculive voids. In these cells we examined the effects of this membrane depolarization on the magnitude of the micturition-related hyperpolarization. In one of these motoneurons, the amplitude of the micturition-related membrane hyperpolarization decreased $3 \mathrm{mV}$ and the motoneuron had depolarized $5 \mathrm{mV}$ between the first and final voids. In the four other motoneurons the magnitude of the micturition-related hyperpolarization was greater in those voids when the membrane potential of the cell had drifted to a more depolarized levcl. In onc of these four cclls, the motoncuron baseline membrane potential had depolarized $12 \mathrm{mV}$ between the time of the first and last void and the micturition-related hyperpolarization was $5 \mathrm{mV}$ larger during the last void.

Figure 2 illustrates the time course of the membrane potential change for five EUS motoneurons during distension-evoked voiding. The membrane was most hyperpolarized when the bladder pressure was greatest and the EUS activity was reduced. Figure 2 also includes data from one animal during the shorterduration isometric micturition contractions (ligated urethra). The pattern of membrane potential change in this motoneuron was similar to the other cells. In 11 of 25 EUS motoneurons, the membrane repolarized to a potential more positive than that immediately preceding the bladder contraction. Action potentials were not observed during this postvoiding repolarization. The apparent "overshoot" during repolarization was detectable in repeated trials in motoneurons where no decrease in the height of the action potential occurred throughout the recording period. This suggests that deterioration of the intracellular recording conditions did not contribute to the repolarized membrane potential levels. In 3 of 11 motoneurons that displayed the more positive repolarization level, we observed a gradual hyperpolarization associated with bladder filling. In the two other mo- 


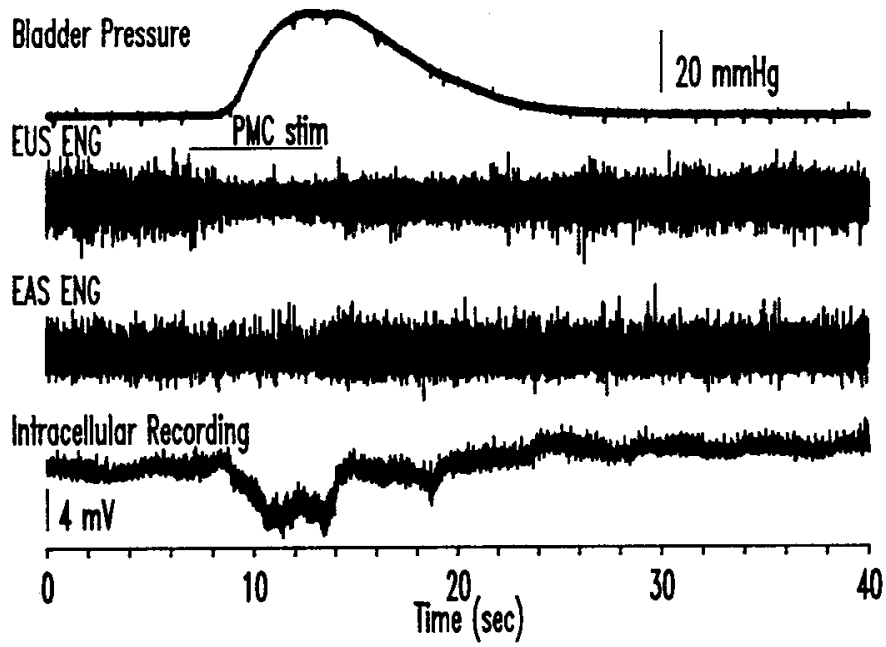

Figure 3. Intracellular recording from an EUS motoneuron during PMC-evoked micturition (resting $E_{m}$, approximately $-50 \mathrm{mV}$; PMC stimulation parameters: $200 \mu \mathrm{A}, 0.5 \mathrm{msec}, 100 \mathrm{~Hz}$ ).

toneurons in which the gradual hyperpolarization was evident, no depolarizing overshoot was observed.

Membrane hyperpolarizations were also seen during PMCevoked micturition. Figure 3 illustrates a typical example in an EUS motoneuron and similar results were obtained in three other EUS motoneurons, each in different animals. The time course and magnitude of the membrane hyperpolarization (5$6 \mathrm{mV}$ ) were similar to those observed during distension-evoked micturition. During PMC-evoked voiding, however, a gradual hyperpolarization was not observed prior to the micturition. Rather, the motoneurons only displayed the rapid and marked hyperpolarization 1-2 sec after the onset of PMC stimulation when micturition began. In addition, these motoneurons did not repolarize to a more positive membrane potential level following micturition as was often observed for distention-evoked micturition.

Four EAS motoneurons recorded in three experiments either depolarized slightly (Fig. 4) or had no change of membrane potential during distension-evoked micturition. One EAS motoneuron recorded during PMC-evoked micturition showed a small depolarization, and the other had no membrane potential change during the micturition.

In seven EUS motoneurons we injected short hyperpolarizing current pulses into the cell during distension-evoked micturition to assess conductance changes in the motoneuron. Conductance changes could be detected by comparing either the peak amplitude or the area of the averaged voltage transients throughout the voiding cycle. Within the same trials, comparison of the area of the averaged voltage transient was found to be a more sensitive indicator of conductance changes, so this method was used throughout this study (see Fig. 5). Area measurements obtained throughout the trial are expressed as percentages of the initial, precontraction value for that trial. Typically, changes in the area of the voltage transient that were $>8 \%$ were detectable with the 12-58 sweeps per average used in the present study. While increasing the number of sweeps per average would have produced smoother averaged records, we maximized our ability to detect the time course of conductance changes during the void by dividing the cycle into more segments containing fewer

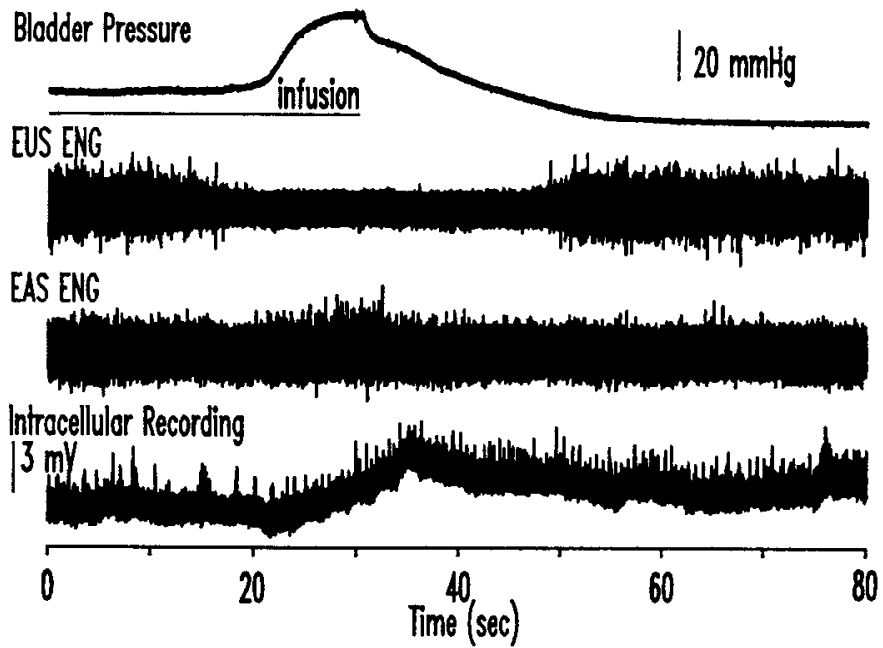

Figure 4. Intracellular recording from an EAS motoneuron during distension-evoked voiding $\left(E_{m}\right.$, about $\left.-50 \mathrm{mV}\right)$. Note the reduction in activity of the EUS but not the EAS ENG during the void.

sweeps. Changes in the area of the averaged voltage deflection were interpreted to be inversely proportional to changes in conductance. In each case the area of voltage transient was significantly reduced during the membrane hyperpolarization associated with the bladder contraction ( $t$ test, $p<0.01$ ), indicating an increase in membrane conductance during this period. The area of the voltage transient was reduced from $21 \%$ to $76 \%$ (average, $45 \%$ ) of the prevoid value within each trial. In all EUS motoneurons tested, the increase in conductance persisted throughout the period of decreased EUS ENG activity during micturition. In five cells the membrane conductance was tested during the postmicturition depolarization. In one cell, the conductance was less than that immediately preceding the void, in another the postvoid conductance was greater (see Fig. 5), and in three cells there were no differences ( $t$ test, $p<0.05$ ).

In three EUS motoneurons large steps of continuous hyperpolarizing current were injected into the cell to test whether membrane hyperpolarization in the absence of micturition produced a conductance change. In each case there was no detectable change in the membrane conductance during the stepwise hyperpolarization $(\leq 12 \mathrm{mV})$ of the motoneuron (not illustrated). It is worth noting that in one of these same motoneurons, the area of the voltage transient decreased to $39 \%$ of control when the membrane hyperpolarized $5 \mathrm{mV}$ during micturition.

The hyperpolarization associated with micturition could be a result of active inhibition or the removal of tonic excitation. To distinguish between these possibilities, records were obtained from five motoneurons using electrodes filled with potassium chloride. In all five motoneurons the membrane hyperpolarization occurring during micturition reversed to a depolarization. This reversal occurred quickly and was seen by the first induced bladder contraction (see Fig. 6), preventing us from obtaining records of the hyperpolarization in these cells prior to its reversal. The magnitude of the membrane depolarization could be influenced by passing continuous depolarizing or hyperpolarizing current through the recording electrode.

\section{Discussion}

It has been shown that EUS and EAS muscles can have different activity patterns during micturition (e.g., Koyanagi et al., 1982; 
A.

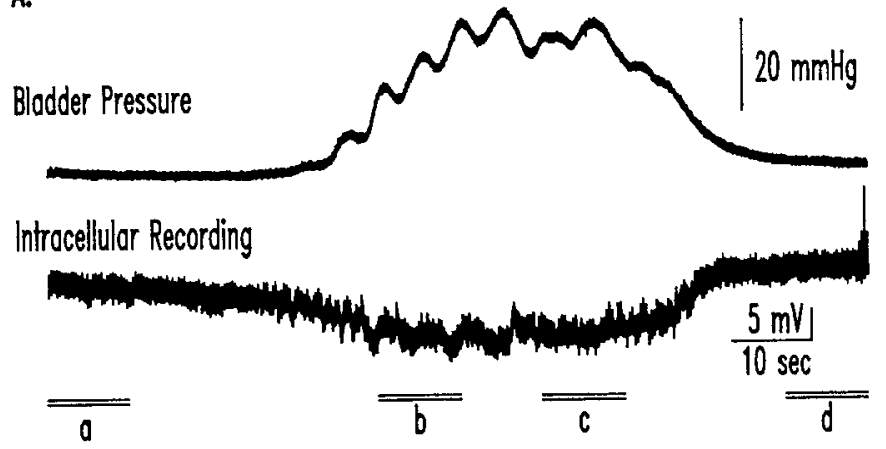

B.
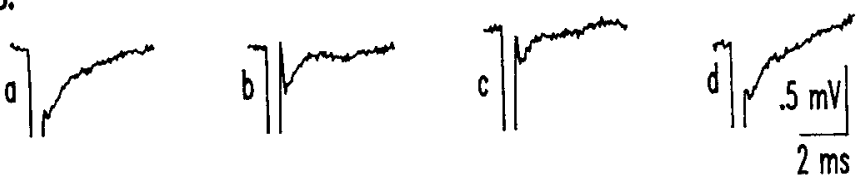

Figure 5. Membrane potential and conductance changes in an EUS motoneuron during an isometric bladder contraction. $A$ shows the bladder pressure trace and intracellular recording from an EUS motoneuron $\left(E_{m}\right.$, approximately $\left.-60 \mathrm{mV}\right) . B$ shows four averages $(29$ sweeps/average) of the $1.5 \mathrm{nA}$ hyperpolarizing pulses $(500 \mu)$ obtained at different times during the trial (marked with the double lines in $A$ ). The area of the average obtained prior to the void was set as $100 \%$, and the area values for averages obtained later in the trial were expressed as percentages of this. The areas of the averages occurring during and immediately following the void were $39 \%, 43 \%$, and $59 \%$, respectively.

Fedirchuk and Shefchyk, 1991). Intracellular recordings from identified EUS and EAS motoneurons obtained in the present study show that the differences in the EUS and EAS activities during evoked micturition can be seen at the level of single motoneurons. EUS motoneurons hyperpolarized during micturition, while EAS motoneurons showed either no membrane potential change or a transient depolarization. Both this study and the earlier study by Shimoda et al. (1992) agree that EUS motoneurons hyperpolarize during distension-evoked micturition. While Shimoda et al. (1992) saw action potentials prior to, and at times during, the voiding cycle, we did not. This difference may be explained by the presence of altered sphincter activity produced by their more rapid bladder filling rates.

The decrease in the voltage transient area is evidence of an increase in EUS motoneuron conductance during the membrane hyperpolarization associated with the isometric bladder contraction (Fig. 5). Previous studies have described voltage-sensitive conductances (i.e., rectifying currents) that could be activated by membrane hyperpolarization (Hochman et al., 1991; Sasaki, 1991). Because we were unable to see comparable conductance increases during step hyperpolarizations of the motoneuron, it seems unlikely that rectifying currents contribute to the conductance increases observed during micturition. Rather, it can be hypothesized that there is an increased inhibitory synaptic input to EUS motoneurons during micturition.

We can only speculate about the mechanisms underlying the gradual hyperpolarization of EUS motoneurons observed during bladder filling. If this slow hyperpolarization is produced by an active conductance, it should be revealed by comparing the conductance before and after micturition. However, we observed that the membrane conductance preceding micturition could be greater, less, or unchanged from that following the

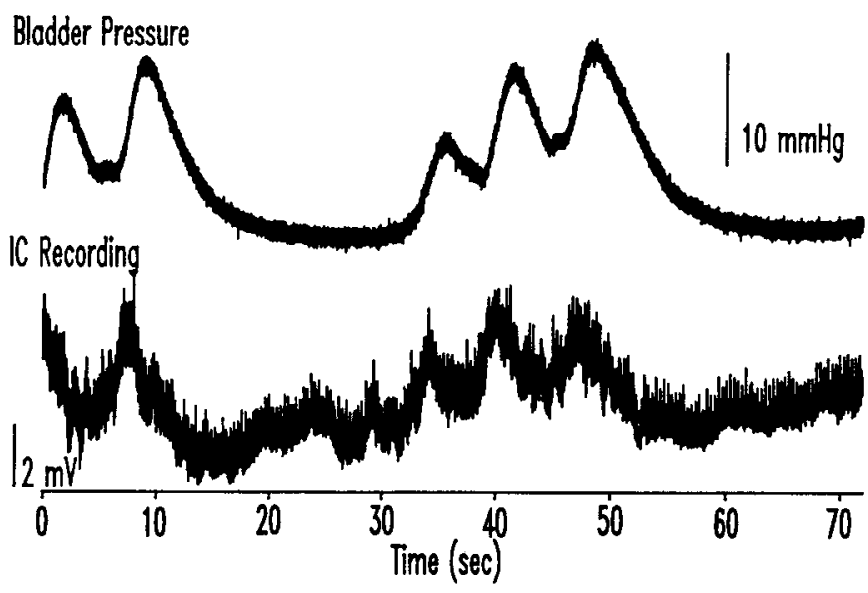

Figure 6. Intracellular $(I C)$ recording from an EUS motoneuron (resting $E_{m}$, approximately $-55 \mathrm{mV}$ ) during an isometric bladder contraction. The hyperpolarization of EUS motoneurons normally associated with the bladder contraction reversed to a depolarization with the use of a chloride-filled microelectrode.

void. The assumption that the conductance measure taken following micturition serves as a good baseline for comparison may be incorrect. At this time micturition-related inhibitory conductances are being turned off and additional excitatory conductances mediating the recovery of EUS activity following micturition may be impinging on the motoneurons.

The reversal of the EUS motoneuron membrane potential hyperpolarization using sodium chloride-filled electrodes implicates a chloride conductance in the production of the hyperpolarization. The membrane potential hyperpolarization normally observed reversed to a depolarization rapidly, often without the passage of current through the microelectrode (Fig. 6). The ease of this reversal and the fact that the conductance changes could be detected from the cell soma suggest that much of the postsynaptic inhibition of the EUS motoneurons during micturition is mediated by synapses located close to, or on, the motoneuron soma (see Burke et al., 1971).

As illustrated in Figure 2, the pattern of EUS motoneuron membrane potential hyperpolarization is very similar during actual voiding and isometric micturition contractions. Often during distension-evoked micturition, the membrane potential repolarized to a level more positive than the value immediately preceding the bladder contraction. The slow membrane hyperpolarization seen prior to micturition (see also Shimoda et al., 1992) would lower the membrane potential by several millivolts and would cause an overestimation of the membrane repolarization following voiding. Since EAS motoneurons do not display this slow hyperpolarization and since rapid filling of the bladder with a large volume of saline solution did not change the motoneuron membrane potential, the slow hyperpolarization seen in some EUS motoneurons is unlikely to be an artifact produced by the physical distension of the bladder. The slow hyperpolarization is more likely due to synaptic actions of reflexes activated by the gradual bladder distension.

In eight EUS motoneurons in five experiments, oscillations of the motoneuron membrane potential were linked to small fluctuations in the bladder pressure (see Figs. 1, 5, 6). Such observations suggest a tight link between the excitation of the parasympathetic efferents to the bladder and the circuitry producing the hyperpolarization of the EUS motoneurons. Also, 
since the change in EUS membrane potential could precede the bladder pressure change (four cases; e.g., see Fig. 6), it is unlikely that the large hyperpolarization associated with the bladder contraction is due to reflexes triggered by the bladder pressure increase itself. As the bladder pressure is a rather indirect reflection of parasympathetic neural output to the bladder, further comparison between the time of onset of the bladder pressure increase and motoneuron membrane potential responses was not done.

Previous studies have used electrical stimulation of the PMC to evoke micturition in the cat (e.g., Shefchyk, 1989; Fedirchuk and Shefchyk, 1991; Mallory et al., 1991). The data from the present study provide evidence that electrical stimulation of the PMC can activate neural circuitry that produces effects in EUS motoneurons comparable to those activated by bladder distension (see Fig. 3). One difference between the responses of EUS motoneurons during distension and PMC-evoked micturition was the slow hyperpolarization preceding the bladder contraction seen only during distension-evoked micturition. If the slow hyperpolarization is due to reflexes produced by bladder filling, the lack of such observations during PMC-evoked micturition is not surprising. In these trials a volume only approximately $50 \%$ of that required to elicit distension-evoked micturition was infused into the bladder and likely did not evoke any reflex effects.

EUS and EAS motoneurons receive similar synaptic input from cutaneous segmental afferent systems (Fedirchuk et al., 1992). The patterns of visceral afferent input to EUS and EAS motoneurons have not yet been described in detail, although rectal distension has been demonstrated to evoke similar responses in both sphincter muscles (Shafik, 1991). The present description of a chloride-mediated postsynaptic inhibition onto EUS motoneurons, but not EAS motoneurons, provides evidence of differential central control of the two sphincter motoneuron populations during micturition. The hyperpolarization of EUS motoneurons demonstrates an action at the motoneuronal level that may contribute to the relaxation of the urcthral sphincter muscle during voiding. In addition, recent work has demonstrated that depolarization of pudendal and perineal primary afferent terminals can occur during micturition (Angel et al., 1992). It is likely that more than one mechanism is responsible for the suppression of excitatory reflexes to the EUS during voiding. The fact that stimulation of the PMC can access the central neural micturition circuitry and produce effects on sphincter motoneurons comparable to those seen during distension-evoked micturition suggests that PMC stimulation will serve as a tool to facilitate future investigations identifying the spinal neurons involved in the neural control of the EUS during micturition.

\section{References}

Angel MJ, McCrea DA, Fyda D, Shefchyk SJ (1992) PAD of perineal afferents evoked by stimulation of sensory afferents and during micturition in the cat. Soc Neurosci Abstr 18:217.19.
Bradley WE, Teague CT (1977) Synaptic events in pudendal motoneurons of the cat. Exp Neurol 56:237-240.

Burke RE, Fedina L, Lundberg A (1971) Spatial synaptic distribution of recurrent and group Ia inhibitory systems in cat spinal motoneurones. J Physiol (Lond) 214:305-326.

Carlen PL, Werman R, Yaari Y (1980) Post-synaptic conductance increase associated with presynaptic inhibition in cat lumbar motoneurones. J Physiol (Lond) 298:539-556.

DeGroat WC (1990) Central control of the lower urinary tract. In: Neurobiology of incontinence, Vol 151 (Bock G, Whelan J, eds), pp 27-56.

Dubrovsky B, Filipini D (1990) Neurobiological aspects of the pelvic floor muscles involved in defecation. Neurosci Biobehav Rev 14:157168.

Fedirchuk B, Shefchyk SJ (1991) Effects of electrical stimulation of the thoracic spinal cord on bladder and external urethral sphincter activity in the decerebrate cat. Exp Brain Res 84:635-642.

Fedirchuk B, Hochman H, Shefchyk SJ (1990) Synaptic inputs onto pudendal motoneurons from sacral segmental and brainstem pathways in the cat. Soc Neurosci Abstr 16:48.10.

Fedirchuk B, Song L, Shefchyk SJ (1991) Investigation of the motoneuronal and premotoneuronal mechanisms of pudendal reflex modulation during micturition in the cat. Soc Neurosci Abstr 17:408.1.

Fedirchuk B, Hochman S, Shefchyk SJ (1992) An intracellular study of perineal and hindlimb afferent inputs onto pudendal motoneurons in the decerebrate cat. Exp Brain Res 89:511-516.

Hochman S, Fedirchuk B, Shefchyk SJ (1991) Membrane electrical properties of external urethral and external anal sphincter somatic motoneurons in the decerebrate cat. Neurosci Lett 127:87-90.

Koyanagi T, Arikado K, Takamatsu T, Tsujui I (1982) Experience with electromyography of the external urethral sphincter in spinal cord injury patients. J Urol 127:271-276.

Kruse MN, Noto H, Roppolo JR, deGroat WC (1990) Pontine control of the urinary bladder and external urethral sphincter in the rat. Brain Res 532:182-190.

Mackel R (1979) Segmental and descending control of the external urethral and anal sphincters in the cat. J Physiol (Lond) 294:105122.

Mallory BS, Roppolo JR, deGroat WC (1991) Pharmacological modulation of the pontine micturition center. Brain Res 546:310-320.

Noto H, Roppolo JR, Steers WD, deGroat WC (1989) Excitatory and inhibitory influences on bladder activity elicited by electrical stimulation in the pontine micturition center in the rat. Brain Res 492: 99-115.

Sasaki M (1991) Membrane properties of external urethral and external anal sphincter motoneurones in the cat. J Physiol (Lond) 440: 345-366.

Shafik A (1991) Dilatation and closing anal reflexes. Acta Anat 142: 293-298.

Shefchyk SJ (1989) The effects of lumbosacral deafferentation on pontine micturition centre-evoked voiding in the decerebrate cat. Neurosci Lett 99:175-180.

Shimoda N, Takakusaki K, Nishizawa O, Tsuchida S, Mori S (1992) The changes in the activity of pudendal motoneurons in relation to refiex micturition evoked in decerebrate cats. Neurosci Lett 135:175178.

Sugaya K, Matsuyama K, Takakusaki K, Mori S (1987) Electrical and chemical stimulations of the pontine micturition center. Neurosci Lett 80:197-201. 